\title{
Immunohistochemical Profile and Distribution of Non-Hodgkin and Hodgkin Lymphoma - An Experience in a Medical College Hospital in Tamil Nadu
}

\begin{abstract}
Context: The subtype distribution of non-Hodgkin lymphoma (NHL) and Hodgkin lymphoma (HL) shows distinct pattern across geographic regions. This study analyzes the subtype distribution of lymphomas based on the WHO 2017 Classification using immunohistochemistry (IHC) and also investigates the IHC profile of various lymphomas. Subjects and Methods: Fifty consecutive cases of lymphoma diagnosed in the department of pathology in a medical college hospital were included in the study. The cases were subcategorized using panels of IHC based on the light microscopic findings. Results: The ratio of NHL to HL was 4.5:1. B-NHL formed 78.5\% $(n=32)$, while T-cell NHL formed $21.5 \%(n=9)$. Among the B-NHL cases, small lymphocytic lymphoma/chronic lymphocytic leukemia (SLL/CLL) (34.4\%) was the most common, followed by diffuse large B-cell lymphoma, NOS (DLBCL NOS) (31.2\%), follicular lymphoma (FL) (25\%), and then by Burkitt's lymphoma (BL) constituting 9.4\%. Peripheral T-cell lymphoma, NOS (PTCL NOS) $(14.1 \%)(n=6)$ was the most common T-cell NHL, followed by angioimmunoblastic T-cell lymphoma (4.8\%), and then by lymphoblastic lymphoma (T-LL) (2.4\%). Among Hodgkin lymphomas, mixed cellularity (MC) accounted for 55.6\% $(n=5)$, lymphocyte depleted type (LD) being $22.2 \%$, while nodular lymphocyte predominant and lymphocyte rich type each constituted $11.1 \%$. Conclusion: SLL/CLL was the most common lymphoma reported in the study followed by DLBCL which is different from the other Indian studies where DLBCL was most common. CD5 expression was noted in $21 \%$ of FLs. PTCL-NOS constituted the most common T-cell lymphoma and MC, the most frequent subtype of classical HL. Further replication studies including larger sample size will be needed to understand the difference in subtype distribution in our population.
\end{abstract}

Keywords: CD5, Hodgkin lymphoma, immunohistochemistry, Lennert's lymphoma, non-Hodgkin lymphoma, small lymphocytic lymphoma

\section{Introduction}

There has been a rising incidence of malignant lymphoma. The incidence varies among the different countries as well as within various regions of a country. ${ }^{[1,2]}$ Age-adjusted incidence of non-Hodgkin lymphoma (NHL) in India is $5 / 100,000{ }^{[1]}$ NHL accounts for $5.1 \%$ of all malignancies and Hodgkin lymphoma (HL) constitutes $9.5 \%$ of cancers in men and $5.5 \%$ in women. ${ }^{[3,4]}$

With the advent of changes in lymphoma therapy, the accurate diagnosis of lymphoma and prognostication wherever possible becomes critical. Similarly, the classification of lymphomas has evolved with the incorporation of various novel immunohistochemical markers in the diagnosis and prognostication. ${ }^{[5-7]}$

\footnotetext{
This is an open access journal, and articles are distributed under the terms of the Creative Commons Attribution-NonCommercialShareAlike 4.0 License, which allows others to remix, tweak, and build upon the work non-commercially, as long as appropriate credit is given and the new creations are licensed under the identical terms.
}

For reprints contact: WKHLRPMedknow_reprints@wolterskluwer.com
Immunohistochemistry (IHC) has become an integral part in the lymphoma diagnosis, histology being the gold standard. The utility of IHC has led to a better understanding of pathogenesis. The panel of IHC markers was chosen based on the morphological findings in light microscopy. The markers include $\mathrm{CD} 45, \mathrm{CD} 20$, and $\mathrm{CD} 3$ to differentiate T- and B-cell lymphomas and CD15 and CD30 for HLs. Other markers used for subtyping were CD10, CD5, CD23, BCL2, Cyclin D1, TdT, ALK, and proliferation marker KI67. There are many studies from the various states of the country reporting on the frequency and distribution of malignant lymphomas. Majority of them are from tertiary referral centers. ${ }^{[8-13]}$

Literature search reveals that the subtype distribution of malignant lymphomas varies

How to cite this article: Shanmugasundaram $\mathrm{S}$, Balan K, Arumugam D. Immunohistochemical profile and distribution of non-Hodgkin and Hodgkin lymphoma - An experience in a medical college hospital in Tamil Nadu. Indian J Med Paediatr Oncol 2020;41:695-701.

\section{Sakthisankari \\ Shanmugasundaram ${ }^{1}$, Krishnagiri Balan², Dhanalakshmi Arumugam $^{2}$}

${ }^{1}$ Department of Pathology, PSG Institute of Medical Sciences and Research, Coimbatore, Tamil Nadu, India, ${ }^{2}$ Department of Pathology, Coimbatore Medical College, Coimbatore, Tamil Nadu, India

Submitted: 06-Mar-2020 Revised: 09-Apr-2020 Accepted: 22-May-2020 Published: 29-Oct-2020

Address for correspondence:

Dr. Sakthisankari

Shanmugasundaram, Department of Pathology, PSG Institute of Medical Sciences and Research, Coimbatore, Tamil Nadu, India.

E-mail: sakthissankari@gmail. com

Access this article online Website: www.ijmpo.org DOI: 10.4103/ijmpo.ijmpo_90_20 Quick Response Code:

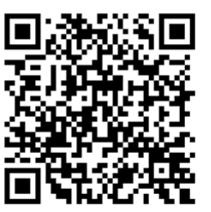


among countries and also across the geographic regions within the countries, the reason for which remains largely unknown. Mondal et al. had reported the frequency of follicular and mantle cell lymphoma to be lower when compared to the Western population. ${ }^{[8]}$ Among HLs, mixed cellularity (MC) was the most common subtype reported by them. ${ }^{[8]}$ DLBCL was the most common B-NHL in most of the Indian studies. ${ }^{[8-13]}$ Arora et al. had found the frequency of peripheral T-cell/NK-cell lymphoma to be higher than Western countries but less than that of the other Asian countries. ${ }^{[9]}$ They had reported peripheral T-cell lymphoma, NOS (PTCL NOS) as the most common T-NHL in contrast to other Indian studies, ${ }^{[10]}$ while Devi et al. had reported lymphoblastic lymphoma (LL) as the most common subtype of T-NHL. ${ }^{[12]}$ Sandhu et al.$^{[1]}$ and Sharma et al. ${ }^{[12]}$ had found that the incidence of follicular and mantle cell lymphoma was lower in India than the USA and Europe. T-cell LL and anaplastic large T/null cell lymphomas are more prevalent in India according to the study by Naresh et al. ${ }^{[13]}$

The study was undertaken with the aim of analyzing the subtype distribution of lymphomas in our population using IHC according to the World Health Organization (WHO) 2017 Classification in the patients visiting the medical college hospital. As a secondary aim, this study also analyzed the immunohistochemical profile of various lymphomas.

\section{Subjects and Methods}

Between August 2011 and August 2013, 50 consecutive cases diagnosed as lymphoma in the histopathology department in a medical college hospital were included in the study. The study was approved by the Institutional Human Ethics committee. Patients of all ages and either gender with both primary and extranodal lymphomas (treatment naive) were included in the study. For histopathological and immunohistochemical studies, the tumor samples were fixed in $10 \%$ neutral buffered formalin, and after tissue processing, they were embedded in a paraffin block. The diagnosis was made by histopathological examination using hematoxylin and eosin. For immunohistochemical studies, $4-\mu \mathrm{m}$ thick sections were taken in specially coated slides using chrome alum and gelatin. Antigen retrieval was done by microwaving. Various panels of markers were chosen based on histological diagnosis. They include CD20, CD3, CD45, CD15, CD30 CD5, CD10, CD23, BCL2, CYCLIN D1, KI67, TDT, and ALK. These antigens are bound with mouse monoclonal antibody (BioGenex) and then detected by the addition of secondary antibody conjugated with horseradish peroxidase polymer and diaminobenzidine substrate. The slides were counterstained with hematoxylin and mounted.

NHL was further categorized morphologically as small-, medium-, or large-cell lymphomas. Accordingly, the panel of markers was chosen as follows.
For HL - CD45, CD20, CD15, CD30, and CD 3.

For NHL small cell type - CD20, CD5, CD23, CD10, CYCLIN D1, and BCL2.

For NHL medium cell type - CD20, CD3, CD5, CD23, CD10, CYCLIN D1, TDT, KI67, and BCL2.

For NHL large cell type-CD20, CD3, CD5, CD23, CD10, CYCLIN D1, TDT, KI67, CD30, ALK, and BCL2.

All the cases were interpreted by experienced pathologists in the field of lymphoma diagnosis.

\section{Results}

During the study period, there were 50 cases of lymphomas which met the inclusion and exclusion criteria. Two cases in which there was no material for IHC workup were excluded from the study. Forty-one cases $(82 \%)$ were diagnosed with NHL and 9 cases $(18 \%)$ were diagnosed with HL.

Of the 41 cases of NHL, $61 \%(n=25)$ were male and $39 \%$ $(n=16)$ were female. Of the nine cases of HL, $55.6 \%$ $(n=5)$ were male and $44.4 \%(n=4)$ were female. In both the groups, males outnumbered females (male-to-female ratio being $1.5: 1)$. About $44 \%(n=22)$ cases were in the age group of $41-60$ years, $32 \%(n=16)$ were $>60$ years, and $24 \%(n=12)$ were $<40$ years.

Nodal lymphomas $(88 \%, n=44)$ were more common than extranodal lymphomas $(12 \%, n=6)$. The extranodal sites included the gastrointestinal tract $(n=2)$, pleura, pericardium, skin, and breast (one each). Cervical lymphadenopathy $(n=26,59.2 \%)$ was the common presentation among the nodal group [Table 1].

B-NHL formed $78.5 \%$ (32 cases), while T-cell NHL formed $21.5 \%$ (9 cases).

Based on the pattern and cellular composition of lymphoma, the lymphomas are classified into small-cell, medium-cell, and large cell type and various panels of immunohistochemical markers were used. The expression of various immunohistochemical markers is tabulated in Table 2.

Small lymphocytic lymphoma/chronic lymphocytic leukemia (SLL/CLL) $(n=11,34.4 \%)$ was the most common B-NHL followed by diffuse large B-cell lymphoma (DLBCL NOS) $(31.5 \%, n=10)$, follicular lymphoma (FL) $(25 \%, n=8)$, and Burkitt's lymphoma (BL) constituting $9.4 \%(n=3)$.

Among the 11 cases of SLL/CLL, peripheral smear findings and bone marrow aspiration studies were available in eight cases. Five cases of SLL/CLL showed bone marrow infiltration, three of which had diffuse infiltration and the remaining two had nodular infiltration. Only three of them showed features of CLL. 


\begin{tabular}{lclc}
\hline \multicolumn{4}{c}{ Table 1: Clinical profile of non-Hodgkin lymphoma } \\
\hline Diagnosis & Number of cases & Nodal/extranodal & $\begin{array}{c}\text { Bone marrow involvement } \\
\text { (number of cases) }\end{array}$ \\
\hline B-NHL & & & 5 \\
SLL & 11 & Nodal & - \\
DLBCL & 9 & 8 nodal and one extranodal (Jejunum) & 3 \\
FL & 9 & Nodal & - \\
BL & 3 & Extranodal & 1 \\
T-NHL & & & - \\
T -lymbhoblastic lymphoma & 1 & Extranodal - pericardium & - \\
Peripheral T cell lymphoma, NOS & 6 & 5 nodal and 1 extranodal (skin) & \\
Angioimmunoblastic T cell lymphoma & 2 & Nodal & \\
\hline
\end{tabular}

NHL: Non-Hodgkin lymphoma, NOS: Not otherwise specified, FL: Follicular lymphoma, SLL: Small lymphocytic lymphoma, DLBCL: Diffuse large B-cell lymphoma, BL: Burkitt's lymphoma

\begin{tabular}{llll}
\hline & \multicolumn{2}{l}{ Table 2: Immunohistochemical profile of B non-Hodgkin lymphoma } \\
\hline Subtype of NHL & Positive markers & Negative markers & Other findings \\
\hline SLL & CD5, CD23 & CD3, CD10, Cyclin & Weak membranous expression of \\
& DD10, CD20, BCL2, Ki67 variable & CD3, Cyclin D1 and & 5 were CD5 positive. \\
FL & & CD5 & 2 cases of Grade III FL showed \\
& & & decreased BCL2 expression \\
DLBCL & BD 20, CD79a, CD10 & CD3, BCL2, TdT & \\
Burkitt lymphoma & CD20, CD10, KI67 95-100\% &
\end{tabular}

SLL: Small lymphocytic lymphoma, DLBCL: Diffuse large B-cell lymphoma, FL: Follicular lymphoma, ALK: Anaplastic lymphoma kinase, NHL: Non-Hodgkin lymphoma

Of the nine cases of FL, one was Grade III. The case showed a predominant follicular pattern $(85 \%-90 \%)$, with a diffuse area $(10 \%-15 \%)$ that was centrocyte rich. There were no sheets of centroblasts in the diffuse areas, and hence, the case was diagnosed as FL Grade III A. Six cases were of Grade II morphology, while the other belonged to Grade I category. Bone marrow involvement was seen in three cases of FL (two were Grade II and the other was Grade III A).

Of the ten cases of DLBCL NOS, nine cases presented with cervical lymphadenopathy, while one case presented as an intestinal mass. Morphological variants included seven cases of centroblastic type, two cases of anaplastic, and one case of immunoblastic category. None of them showed bone marrow involvement at diagnosis.

All the three cases of BL were extranodal lymphomas, of which one case presented with intussusception in a 3-year old child. There was also a rare case of BL presented with breast mass.

PTCL-NOS $(14.1 \%, n=6)$ was the most frequent T-cell NHL, followed by angioimmunoblastic T-cell lymphoma (AITL) $(4.8 \%, n=2)$, T-LL constituting 2.4\% $(n=1)$. All except one case of PTCL NOS presented with nodal enlargement. LL presented with a pericardial mass.

Among HLs, MC formed 55.6\% $(n=5)$, lymphocytedepleted type (LD) formed 22.2\% $(n=2)$, and nodular lymphocyte predominant HL (NLPHL) and lymphocyte rich (LR) type each constituted $11.1 \%(n=1)$. The age of the patients ranged from 19 to 78 years. All the nine patients were seronegative for HIV. Both the cases of HL, LD, occurred in elderly males.

\section{Immunohistochemical analysis}

All the 11 cases of SLL/CLL were CD5 and CD23 positive with weak expression of CD20 [Figure 1].

Of the 10 cases of DLBCL, CD10 positivity was noted in $5(50 \%)$ cases [Figure 2]. All the cases showed diffuse expression of CD20 and CD79a. Ki67 index varied from 76 to $90 \%$.

All the cases of FL expressed CD10, BCL2, and CD20. Grade III FL showed less intense CD10 expression. Three of the nine cases of FL showed positivity for CD5.

All the cases of T-NHL expressed CD3. Ki67 was a variable ranging from $20 \%$ to $90 \%$.

Eight cases of classic HL expressed CD30, of which five cases also expressed CD15 [Figure 3].

One case of NLPHL was positive for CD20 and negative for both CD15 and CD30.The background polymorphous cells showed a variable expression of CD3 and CD20.

\section{Discussion}

Geographic variations in the incidence as well as distribution of histological subtypes of lymphoma are 


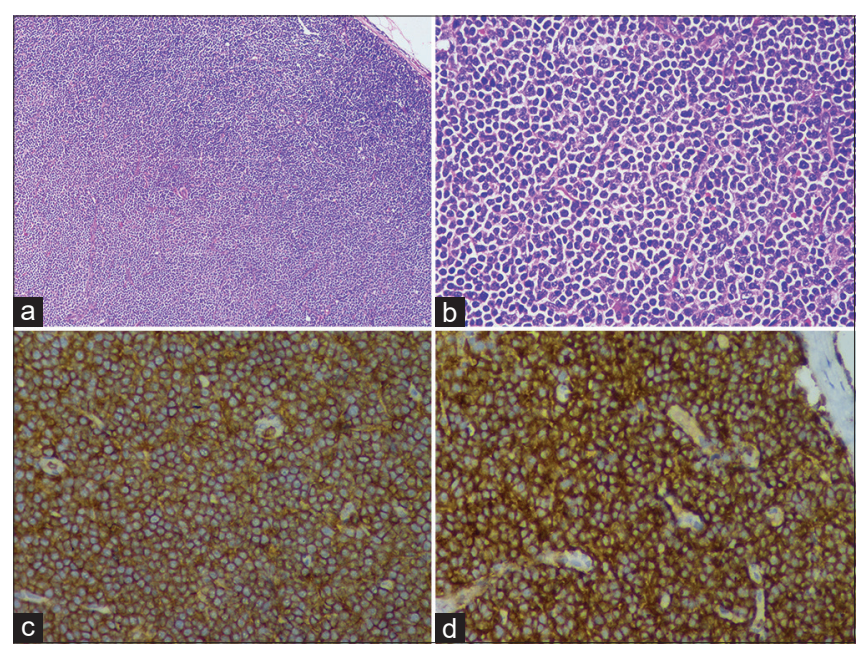

Figure 1: (a) Small lymphocytic lymphoma/chronic lymphocytic leukemia sheets of small lymphoid cells replacing lymph node $(H$ and $E, \times 10)$. (b) Cells with scant cytoplasm and coarse chromatin of nucleus $(H$ and $E, \times 40)$. (c) Cells expressing CD5 (Immunohistochemistry, $\times 40$ ). (d) Cells expressing CD23 (Immunohistochemistry, $\times 40$ )

well-documented. A higher rate of nasal T-cell lymphomas has been reported in China. BL occurs in endemic form in Africa. Gastric lymphomas and adult T-cell leukemias are reported to be more frequent in Italy and Japan, respectively. ${ }^{[1]}$ The incidence rates for NHL in India are quite high in urban cities when compared to rural centers. ${ }^{[1]}$ The incidence rate is one-quarter of what is reported in America and Europe. ${ }^{[1]}$

The male-to-female ratio is 1.6 in Asia compared to 1.2 and 1.1 in North America and Europe, respectively. The present study also reports a male preponderance. The median age was 50 years which is higher when compared to Eastern India in which the median age was 39.4 years. ${ }^{[8]}$

The ratio of NHL to HL was 4.5:1 which is higher when compared to another South Indian study which had reported the ratio of NHL to $\mathrm{HL}$ to be $3.6: 1 .^{[9]}$

In line with most other studies in India, the most common mode of presentation in the present study was lymphadenopathy constituting to $86 \% .^{[8-11]}$ The most commonly involved lymph node groups were cervical $(n=22,59.5 \%)$ followed by inguinal $(18.2 \%, \mathrm{n}=8)$ and axillary $(11.3 \%, \mathrm{n}=5)$, Which is similar to that reported by Devi et al. ${ }^{[11]}$ Extranodal presentation was seen in $14 \%$ of cases in the present study, the frequency of which was much lower than that reported in Northern and Eastern India (44.2 and $27.7 \%$, respectively). ${ }^{[10,11]}$ This could be attributed to the smaller sample size of the present study.

The extranodal lymphomas in the present study were exclusively Non-Hodgkin's lymphomas. The predominant subtype was BL which constituted for about $50 \%$, which is in contrast to the other regions of India and other Asian countries where DLBCL was the most frequent subtype. ${ }^{[1-13]}$ The gastrointestinal tract was the most

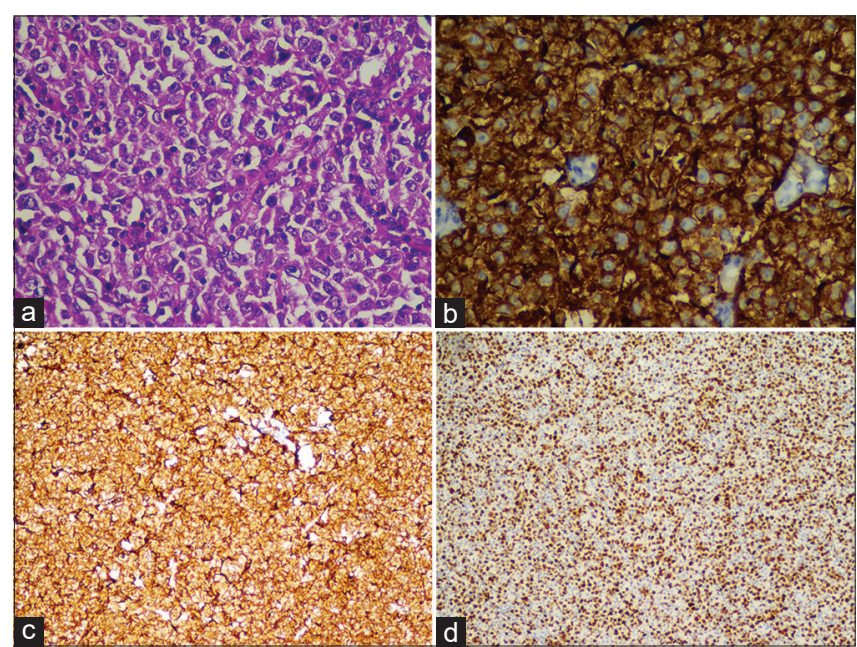

Figure 2: (a) Diffuse large B-cell lymphoma - Sheets of large cells with prominent nucleoli, (H and E, $\times 40)$. (b) Large cells with strong membranous expression of CD20 (Immunohistochemistry, $\times 40$ ). (c) Large cells expressing CD10 (Immunohistochemistry, $\times 10$ ). (d) High proliferation index with Ki67 staining (Immunohistochemistry, $\times 10)$

common site for extranodal lymphomas which is consistent with other Indian studies. ${ }^{[12-14]}$ [Table 3].

Bone marrow involvement was seen in $22 \%(n=9)$ of NHL. The reported percentage varied from 20 to $40 \%$ in various western studies and $30.3 \%-63 \%$ in Indian studies. ${ }^{[8-10]}$ Bone marrow biopsy studies were available only in 28 cases.

\section{B-cell non-Hodgkin lymphoma}

The ratio between B-cell and T-cell NHL was 3.5:1. SLL formed the major proportion of B-cell NHL constituting $34.4 \%$ in contrast to most other studies where DLBCL NOS was the most frequent lymphoma. ${ }^{[15-17]}$ This could be possibly due to higher number of elderly patients found in our study. Extensive literature search did not find any Indian study reporting SLL/CLL to be more frequent. ${ }^{[11-17]}$ Currently, available studies on the distribution of lymphomas are from tertiary referral centers where aggressive lymphomas are likely to be referred. Our study population being the patients attending a teaching hospital, they can show a distribution much different from those studies. However, one cancer registry-based European study done by Marcos et al., which had analyzed 5-year survival rate in patients with lymphoid neoplasms, had observed a greater number of SLL/CLL cases during their study period of 7 years..$^{[18]}$ All the 11 cases of SLL showed weak expression of CD20 similar to that observed by Veronika et al. in which it was shown that of all mature B-cell neoplasms, SLL/CLL has the dim expression of CD20. ${ }^{[17]}$

SLL/CLL was followed in frequency by DLBCL constituting to $28 \%$, which is in contrast to majority of other Indian studies reporting the incidence to be between $30 \%$ and 58\%. ${ }^{[10-14,17]}$ However, Roy et al. and Kalyan 


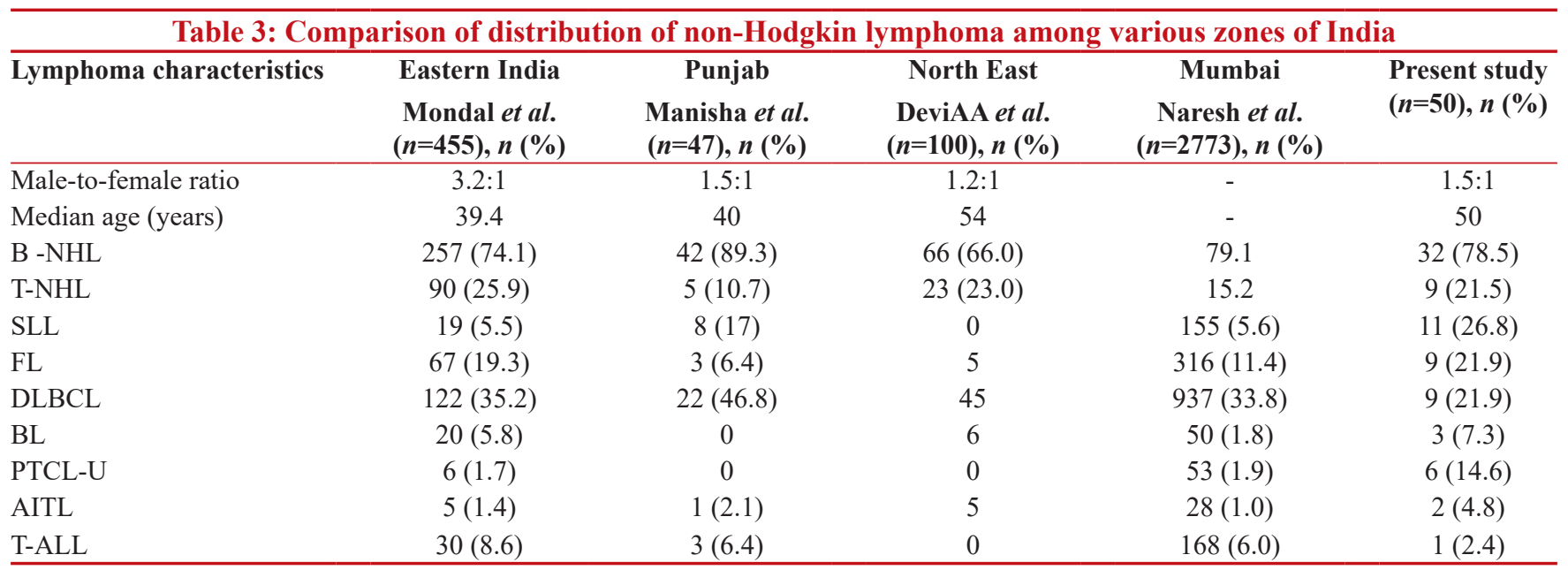

NHL: Non-Hodgkin lymphoma, AITL: Angioimmunoblastic T-cell lymphoma, PTCL: Peripheral T-cell lymphoma, BL: Burkitt's lymphoma, DLBCL: Diffuse large B-cell lymphoma, FL: Follicular lymphoma, SLL: Small lymphocytic lymphoma, T-ALL: T-cell acute lymphoblastic leukemia

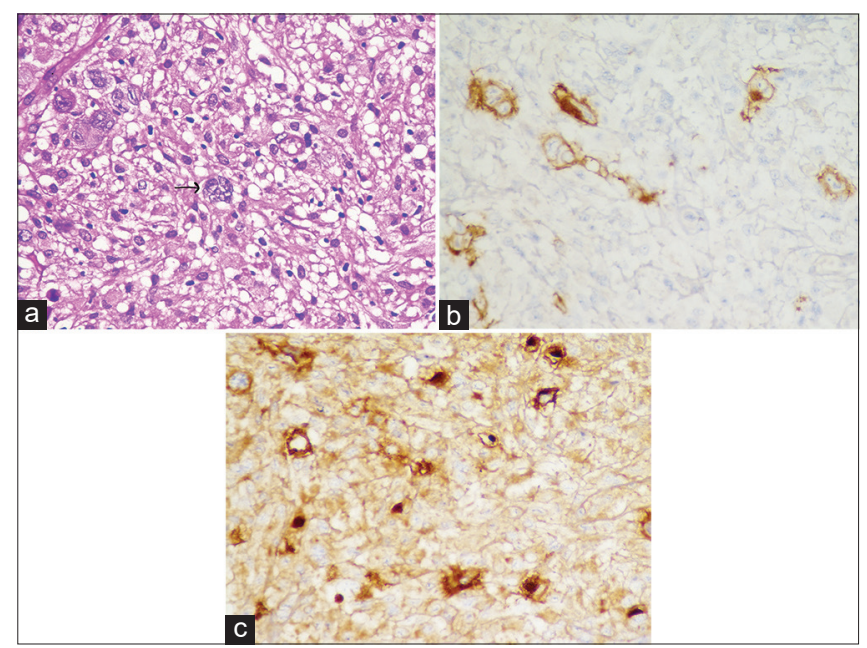

Figure 3: (a) Pleomorphic Reed-Sternberg cells (H and E, ×40). (b) RS cells expressing CD30 (Immunohistochemistry, $\times 10$ ). (c) RS cells expressing CD15 (Immunohistochemistry, ×10)

et al. had reported the incidence to be $29.3 \%$ and $26 \%$, respectively, ${ }^{[19,20]}$ which were not much different from the present study.

DLBCL NOS was the most common of aggressive NHL observed in the current study. The most common mode of presentation was cervical lymphadenopathy except one which presented with an intestinal lymphoma. None of them showed any bone marrow involvement at diagnosis. In contrast, bone marrow involvement had been reported to be varying from 4 to $20 \%$ in the Indian population. ${ }^{[21]} \mathrm{CD} 10$ positivity was noted in $5(50 \%)$ cases which is similar to that reported by Diwivedi et al. ${ }^{[22]}$

Among the nine cases of FLs, we observed five of them to express CD5 constituting to $21 \%$ of CD5-positive lymphomas in our study which was similar to the observation made by Dong et al., in which FL accounted for $24 \%$ of cases positive for both CD5 and CD10. ${ }^{[23]}$ The case of Grade III FL showed decreased expression of BCL2 which is similar to the other studies. ${ }^{[24,25]}$

All the three cases of BL were extranodal lymphomas, of which one case presented with intussusception in a 3-yearold child. The presentation was similar to other studies which had shown abdomen, particularly the ileocecal region as the most common site of involvement. ${ }^{[14]}$ We also found an unusual presentation of BL with breast lump in a 25-year-old female. Histology showed that the typical morphology of BL and Ki67 proliferation index was 97\%. Primary BL of the breast is much less common than the other types of lymphoma. Primary breast lymphomas are extremely rare, and the most common histologic type is DLBCL. ${ }^{[26]}$

\section{T-cell non-Hodgkin lymphoma}

T-NHL constituted for $24 \%(n=9)$ which is similar to other Indian studies. ${ }^{[11-17,27]}$ The frequency of T-NHL is higher in India when compared to Western countries but less than what is reported in other East Asian countries. PTCL NOS was the most common subtype of T-NHL in the present study similar to that reported by Arora et al. and Nair et al..$^{[9,28]}$ Sharma et al. had reported T-LL to be more common followed by anaplastic large cell lymphoma (ALCL) and AITL accounting for 6.4, 2.1, and 2.1\% of all NHL, respectively. ${ }^{[27]}$ Naresh et al. had reported the incidence of T-LL and ALCL to be $6 \%$ and $4.3 \%$, respectively. ${ }^{[13]}$ Although ALCL was the most common PTCL in Eastern India, it was not identified in our study, the reason for which is unknown.

We also found a rare variant of PTCL, Lennert's lymphoma in a patient who presented with bilateral cervical lymphadenopathy. Morphologically, it showed microgranulomas typical of the lymphoepithelioid lymphoma. All the neoplastic cells expressed CD3. 


\section{Hodgkin lymphoma}

HL included eight cases of classic HL and one case of NLPHL HL. The mean age of presentation in HL was 34 years which is almost similar to studies in South and Eastern India where the mean age reported was 33 and 31.3 years, respectively. ${ }^{[8,9]}$ All the cases of HL presented with peripheral lymphadenopathy. Extranodal presentation and bone marrow involvement were not seen in any of these cases. The other studies in India too have not reported any extranodal presentation. In contrast, bone marrow involvement was reported to be $14.3 \%$ in HL. ${ }^{[8,9]}$

The most common subtype found in the study was MC ( $n=5,55.6 \%)$, followed by LD. Reed-Sternberg cells in LD HL showed strong expression of both CD15 and CD30 [Figure 2]. Other Indian studies had also reported $\mathrm{MC}$ to be the most common subtype of HL, followed by nodular sclerosis subtype, which is in contrast to Western countries where NS is much more frequent than other subtypes. ${ }^{[29,30]}$ LD subtype was found in elderly adults.

One case each of LR and NLPHL HL were identified in this study. CD15 expression was seen in 5 (62.1\%) of eight cases of classic HL. This is slightly higher than reported in a study which has reported that $49.11 \%$ of the cases expressed CD15. ${ }^{[29]}$

\section{Limitations}

One of the limitations of the study is a smaller sample size, yet there was a significant difference in subtype distribution. Follow-up data were not available to assess the value of prognostic markers used in the IHC panel.

\section{Conclusion}

The distribution of lymphoma subtypes differs from other regions of India. SLL/CLL was the most common NHL reported in our study, followed by DLBCL which differs from other studies. About $21 \%$ of FL expressed CD5 and CD10. PTCL NOS was the most common T-NHL in the study. MC was the most frequent HL. CD15 expression was more frequent in HL. IHC is an essential diagnostic aid in categorization. Further replication studies including larger samples are essential to understand the difference in subtype distribution in our population.

\section{Financial support and sponsorship}

Nil.

\section{Conflicts of interest}

There are no conflicts of interest.

\section{References}

1. Nair R, Arora N, Mallath MK. Epidemiology of non-Hodgkin's lymphoma in India. Oncology 2016;91 Suppl 1:18-25.

2. Boffetta P. Epidemiology of adult non-Hodgkin lymphoma. Ann Oncol 2011;22:27-31.
3. Yeole BB. Trends in the incidence of non-Hodgkin's lymphoma in India. Asian Pac J Cancer Prev 2008;9:433-6.

4. Parkin D M, Bray F, Ferlay J. Global cancer statistics. Cancer J Clin 2005;55:74-108.

5. Morton LM. Dissecting lymphoma incidence to inform epidemiologic and clinical research. Leuk Lymphoma 2013;54:1575-6.

6. Chan JK. The new World Health Organization classification of lymphomas: The past, the present and the future. Hematol Oncol 2001;19:129-50.

7. Swerdlow SH, Campo E, Harris NL, Jaffe ES, Pilleri SA, Stein $\mathrm{H}$, et al.., editors. World Health Organization Classification of Tumours of Haematopoietic and Lymphoid Tissue. Lyon: IARC Press; 2008.

8. Mondal SK, Mandal PK, Samanta TK, Chakaborty S, Roy SD, Roy S. Malignant lymphoma in Eastern India: A retrospective analysis of 455 cases according to World Health Organization classification. Indian J Med Paediatr Oncol 2013;34:242-6.

9. Arora N, Manipadam MT, Nair S. Frequency and distribution of lymphoma types in a tertiary care hospital in South India: Analysis of 5115 cases using the World Health Organization 2008 classification and comparison with world literature. Leuk Lymphoma 2013;54:1004-11.

10. Sandhu DS, Sharma A, Kumar L. Non-Hodgkin's lymphoma in Northern India: An analysis of clinical features of 241 cases. Indian J Med Paediatr Oncol 2018;39:42-5.

11. Devi AA, Sharma TD, Singh YI, Sonia H. Clinicopathological profile of patients with non-hodgkin's lymphoma at a regional cancer center in Northeast India. J Sci Soc 2017;44:140-4.

12. Sharma M, Mannan R, Madhukar M, Navani S, Manjari M, Bhasin TS, et al. Immunohistochemical (IHC) analysis of nonHodgkin's Lymphoma (NHL) spectrum according to WHO/ REAL classification: A single centre experience from Punjab, India. J Clin Diagn Res 2014;8:46-9.

13. Naresh KN, Srinivas V, Soman CS. Distribution of various subtypes of nonHodgkin's lymphoma in India: A study of 2773 lymphomas using R.E.A.L. And WHO classifications. Ann Oncol 2000;11:637.

14. Chakrabarti S, Sarkar S, Goswami BK, Mondal S, Roy A, Das S. Hodgkin's and Non-Hodgkin's lymphomas in an Indian rural medical institution: Comparative clinicopathologic analysis. Asian Pac J Cancer Prev 2010;11:1605-8.

15. Mushtaq S, Akhtar N, Jamal S, Mamoon N, Khadim T, Sarfaraz $\mathrm{T}$, et al. Malignant lymphomas in Pakistan according to the WHO classification of lymphoid neoplasms. Asian Pac J Cancer Prev 2008;9:229-32.

16. Prakash G, Sharma A, Raina V, Kumar L, Sharma MC, Mohanti BK, et al. B cell non-Hodgkin's lymphoma: Experience from a tertiary care cancer center. Ann Hematol 2012;91:160311.

17. Prevodnik VK, Lavrenčak J, Horvat M, Novakovič BJ. The predictive significance of CD20 expression in B-cell lymphomas. Diagn Pathol 2011;6:33.

18. Gragera MR, Allemani C, Tereanu C, De Angelis R, Capocaccia R, Maynadie M, et al. HAEMACARE Working Group (2011) Survival of European patients diagnosed with lymphoid neoplasms in 2000-2002: Results of the HAEMACARE project. Haematologica 2011;96:720-8.

19. Roy A, Kar R, Basu D, Badhe BA. Spectrum of histopathologic diagnosis of lymph node biopsies: A descriptive study from a tertiary care center in South India over 5 $1 \frac{1}{2}$ years. Indian J Pathol Microbiol 2013;56:103-8.

20. Kalyan K, Basu D, Soundararaghavan J. Immunohistochemical typing of non-Hodgkin's lymphoma-comparing working 
formulation and WHO classification. Indian J Pathol Microbiol 2006;49:203-7.

21. Babu SM

Kuntegowdanaha lymphoma: A retrospective study from a regional care center in South India. Indian J Cancer 2018;55:66-9.

22. Dwivedi A, Mehta A, Solanki P. Evaluation of immunohistochemical subtypes in diffuse large B-cell lymphoma and its impact on survival. Indian J Pathol Microbiol 2015;58:453-8.

23. Dong HY, Gorczyca W, Liu Z, Tsang P, Wu CD, Cohen P, et al. B-cell lymphomas with coexpression of CD5 and CD10. Am J Clin Pathol 2003;119:218-30.

24. Rao IS. Role of immunohistochemistry in lymphoma. Indian J Med Paediatr Oncol 2010;31:145-7.

25. Higgins RA, Blankenship JE, Kinney MC. Application of immunohistochemistry in the diagnosis of non-Hodgkin and Hodgkin lymphoma. Arch Pathol Lab Med 2008;132:441-61.
26. Janbabai G, Kayedimajd S, Alian S, Naghshvar F, Rashidi M, Farazmandfar T. Bilateral breast swelling in a 23-year-old woman with Burkitt lymphoma. J Res Med Sci 2012;17:1188-91.

27. Lakshmanan A, Sikri D, Patil S, Kurian A, Annapurneswari S, Nair S. Frequency, distribution, and immunomorphologic characteristics of peripheral T-cell lymphoma, not otherwise specified in a tertiary care center in Southern India. Indian J Pathol Microbiol 2018;61:204-8.

28. Nair RA, Vasudevan JA, Jacob PM, Sukumaran R. Profi ling of peripheralT-cell lymphomas in Kerala, South India: A 5-year study. Indian J Pathol Microbiol 2017;60:206-8.

29. Bidyut Krishna Goswami BK, Sarkar S, Chakrabarti S, Mondal S, Roy A, Deb AR. Clinico-pathologic profile of Hodgkin's lymphoma in a rural medical college. Indian $\mathrm{J}$. Hematol. Blood Transfus 2008;24:166-9.

30. Patkar N, Mehta J, Kulkarni B, Pande R, Advani S, Borges A. Immunoprofile of Hodgkin's lymphoma in India. Indian J Cancer 2008;45:59-63. 